TRANSACTIONS OF THE

AMERICAN MATHEMATICAL SOCIETY

Volume 275, Number 1, January 1983

\title{
DIMENSION OF STRATIFIABLE SPACES
}

BY

SHINPEI OKA

\begin{abstract}
We define a subclass, denoted by $E M_{3}$, of the class of stratifiable spaces, and obtain several dimension theoretical results for $E M_{3}$ including the coincidence theorem for $\operatorname{dim}$ and Ind. The class $E M_{3}$ is countably productive, hereditary, preserved under closed maps and, furthermore, the largest subclass of stratifiable spaces in which a harmonious dimension theory can be established.
\end{abstract}

1. Introduction. Beyond metric spaces, the following line of generalized metric spaces has been established by many authors $[\mathbf{S}, \mathbf{C}, \mathbf{B}, \mathbf{H}, \mathbf{O k}]$ :

$$
\text { metric } \rightarrow \text { Lašnev }^{1} \rightarrow M_{1} \rightarrow \text { stratifiable } \rightarrow \text { paracompact } \sigma .
$$

After Katětov and Morita's work for metric spaces, the first attack to this line in dimension theory was done by Leibo $\left[\mathbf{L}_{1}\right]$ who proved the equality $\operatorname{dim} X=\operatorname{Ind} X$ for any Lašnev space $X$. Nagami extended this result by defining $L$-spaces $\left[\mathrm{N}_{\mathbf{3}}\right]$ and free $L$-spaces $\left[\mathbf{N}_{4}\right]$. Free $L$-spaces form a countably productive and hereditary class containing every Lašnev space and included in the class of $M_{1}$-spaces. It is now desired to develop a satisfactory dimension theory of a still larger class of generalized metric spaces, say, $M_{1}$-spaces or stratifiable spaces.

In this direction we define a subclass of stratifiable spaces in terms of a special kind of $\sigma$-closure-preserving collection.

Definition 1.1. Let $X$ be a space. A collection $\mathcal{E}$ of subsets of $X$ is called an encircling net (or, for short, E-net) if for any point $x$ and any open neighborhood $U$ of $x$, there exists a subcollection $\mathscr{F}$ of $\mathcal{E}$ such that $x \in X-\mathscr{F}^{*} \subset U$ and $\mathscr{F}^{*}$ is a closed set of $X$ (where $\mathscr{F}^{*}$ denotes the union of the members of $\mathscr{F}$ ).

By $E_{3}$ we denote the class of stratifiable spaces with $\sigma$-closure-preserving E-nets, and by $M_{3}$ the class of stratifiable spaces.

The class $E M_{3}$ is countably productive, hereditary and preserved under closed maps as well as perfect maps (Corollary 3.9).

Our first main result is a characterization of members of $E M_{3}$ as those spaces which are the perfect (closed) images of zero-dimensional stratifiable spaces (Theorem 3.8). This means that $E M_{3}$ is just the maximal perfect subclass of $M_{3}$ in the sense of Nagami $\left[\mathbf{N}_{1}\right]$.

Received by the editors May 15, 1981 and, in revised form, December 1, 1981.

1980 Mathematics Subject Classification. Primary 54E20, 54F45; Secondary 54E18.

$K e y$ words and phrases. Stratifiable space, $\sigma$-space, $E$-net, $L E$-net, $E$-quartet, $E$-map, fiber product.

1 A space is called a Lašnev space if it is the closed image of a metric space.

(C) 1983 American Mathematical Society $0002-9947 / 82 / 0000-0447 / \$ 02.75$ 
The second main results appear in Theorems 4.2 and 4.3 and consist of the following theorems for $E M_{3}$ :

(a) the equidimensional $G_{\delta}$-envelope theorem,

(b) the dimension raising theorem, ${ }^{2}$

(c) the decomposition theorem,

(d) the coincidence theorem for dim and Ind.

These theorems for $E M_{3}$ extend the corresponding theorems for free $L$-spaces $\left[\mathbf{N}_{4}\right]$ as well as those for Lašnev spaces $\left[\mathbf{L}_{1}, \mathbf{L}_{2}, \mathbf{O}_{1}\right]$.

It is an open problem whether the inclusion $E M_{3} \subset M_{3}$ is proper. But the characterization above implies that $E M_{3}$ is the largest ${ }^{3}$ subclass of $M_{3}$ in which the dimension raising theorem holds. We also see in Corollary 4.5 that $E M_{3}$ is the largest ${ }^{3}$ subclass of $M_{3}$ in which the decomposition theorem and the equidimensional $G_{\delta}$-envelope theorem simultaneously hold.

Our arguments are based on Gruenhage and Junnila's result that a stratifiable space is an $M_{2}$-space [G, J]. Indeed, though we use the word "stratifiable" in view of its significance, what we need is only the existence of a $\sigma$-closure-preserving quasi-base.

Conventions. Throughout this paper a space is a Hausdorff topological space, and a map means an onto continuous one. Let $X, Y$ be spaces and let $f: X \rightarrow Y$ be a map. For a collection $\mathscr{F}$ of subsets of $X$, the symbol $\mathscr{F}^{*}$ denotes the union of all members of $\mathscr{F}$, and $f(\mathscr{F})$ means the collection of subsets of $Y$ of the form $\{f(F): F \in \mathscr{F}\}$. For a subset $Z$ of $X$ we denote by $\bar{Z}$ (or $\mathrm{Cl} Z$ ) the closure of $Z$, by Int $Z$ the interior of $Z$, and by $B d Z$ the boundary of $Z$.

2. Encircling nets and large encircling nets. Encircling nets are naturally strengthened as follows:

Definition 2.1. Let $X$ be a space. A collection $\mathcal{E}$ of subsets of $X$ is called a large encircling net (or, simply, an $L E$-net) if for any disjoint closed sets $C$ and $K$ of $X$, there exists a subcollection $\mathscr{F}$ of $\mathcal{E}$ such that $C \subset \mathcal{F}^{*} \subset X-K$ and $\mathcal{F}^{*}$ is a closed set of $X$.

REMARKS. Since an $L E$-net is a net in the usual sense, it follows from Siwiec-Nagata [SN] that a space with a $\sigma$-closure-preserving $L E$-net is a $\sigma$-space. But a space with a $\sigma$-closure-preserving $E$-net is not necessarily a $\sigma$-space as will be seen in Example 2.8 . On the other hand it is trivial that a regular $\sigma$-space $X$ with ind $X \leqslant 0$ admits a $\sigma$-closure-preserving $E$-net, and that a normal $\sigma$-space $X$ with $\operatorname{dim} X \leqslant 0$ admits a $\sigma$-closure-preserving $L E$-net.

Proposition 2.2. A metric space admits a $\sigma$-locally finite LE-net.

Proof. Let $M$ be a metric spaces and $\left\{\mathcal{E}_{i}: i=1,2, \ldots\right\}$ a sequence of locally finite closed covers of $M$ such that, for each $i$, the diameter of each member of $\mathcal{E}_{i}$ is smaller than $1 / i$. Let $C, K$ be disjoint closed sets of $M$ and put

$$
\mathscr{F}_{i}=\left\{E \in \mathcal{E}_{i}: E \cap C \neq \varnothing \text { and } E \cap K=\varnothing\right\} .
$$

\footnotetext{
2 The dimension raising theorem for a topological class $\mathcal{C}$ is: If $X \in \mathcal{C}$ and $\operatorname{dim} X \leqslant n$, then $X$ is the image of a space $X_{0} \in \mathcal{C}$ with $\operatorname{dim} X_{0} \leqslant 0$ under a perfect map of order not greater than $n+1$.

${ }^{3}$ When using this word we take no account of infinite-dimensional spaces in the sense of dim.
} 
It is then clear that $\cup_{i=1}^{\infty} \mathscr{F}_{i}^{*}$ is a closed set of $X$ including $C$ but not meeting $K$. Hence $\cup_{i=1}^{\infty} \mathcal{E}_{i}$ is a $\sigma$-locally finite $L E$-net on $M$, which completes the proof.

PROPOSITION 2.3. The property of having a $\sigma$-closure-preserving LE-net is preserved under closed maps.

We thus have

Proposition 2.4. A Lašnev space admits a $\sigma$-closure-preserving LE-net, and hence it is a member of $E M_{3}$.

LEMMA 2.5. If $\mathcal{E}$ is an E-net (resp. LE-net) on a space, then $\{\bar{E}: E \in \mathcal{E}\}$ is an E-net (resp. LE-net) on the space.

Proposition 2.6. The property of having a o-closure-preserving E-net is countably productive, hereditary and preserved under perfect maps.

Proof. Let $X_{i}, i=1,2, \ldots$, be spaces with $\sigma$-closure-preserving $E$-nets $\mathcal{E}_{i}$. It is then clear that

$$
\left\{E_{j} \times \prod_{i=1, i \neq j}^{\infty} X_{i}: E_{j} \in \mathcal{E}_{j}, j=1,2, \ldots\right\}
$$

is a $\sigma$-closure-preserving $E$-net on $\prod_{i=1}^{\infty} X_{i}$.

By the preceding lemma it is obvious that the property is hereditary.

Let $X$ be a space with a $\sigma$-closure-preserving $E$-net $\mathcal{E}$ and let $f: X \rightarrow Y$ be a perfect map onto a space $Y$. By Lemma 2.5 we may assume that every finite intersection of members of $\mathcal{E}$ is again a member of $\mathcal{E}$. To show that $f(\mathcal{E})$ is an $E$-net on $Y$ let $y \in Y$ and let $U$ be an open neighborhood of $y$. There exist subcollections $\mathcal{E}_{i}, 1 \leqslant i \leqslant k$, of $\mathcal{E}$ such that $f^{-1}(y) \subset X-\bigcap_{i=1}^{k} \mathcal{E}_{i}^{*} \subset f^{-1}(U)$ and $\mathcal{E}_{i}^{*}$ is a closed set of $X$. It then follows from assumption that $f\left(\cap_{i=1}^{k} \mathcal{E}_{i}^{*}\right)$ is a closed set of $Y$ written as a union of members of $f(\mathcal{E})$ such that $y \in Y-f\left(\cap_{i=1}^{k} \mathcal{E}_{i}^{*}\right) \subset U$. This completes the proof.

Proposition 2.7. Let $X$ be a space (resp. a semistratifiable space). Then the following statements are equivalent:

(1) X admits a o-closure-preserving LE-net (resp. E-net).

(2) $X$ admits a $\sigma$-locally finite LE-net (resp. E-net).

(3) X admits a o-discrete LE-net (resp. E-net).

Proof. It follows from Lemma 2.5 and a remark above that a space with a $\sigma$-closure-preserving $L E$-net admits a $\sigma$-closure-preserving net of closed sets, and therefore it is semistratifiable. Hence the proposition is immediate from Lemma 2.5 and the following fact, which is essentially due to Siwiec and Nagata [SN]: Let $X$ be a semistratifiable space and $\mathcal{E}$ a $\sigma$-closure-preserving collection of closed sets of $X$. Then there exists a $\sigma$-discrete collection $\mathscr{F}$ of closed sets of $X$ such that each member of $\mathcal{E}$ is a union of members of $\mathcal{F}$.

As for famous pathological spaces, we have the following results which imply particularly that the existence of $\sigma$-closure-preserving $E$-nets does not mean, in general, that of $\sigma$-closure-preserving $L E$-nets (but, for stratifiable spaces, the former means the latter as will be seen in Theorem 3.8). 
EXAMPLES 2.8. (1) The Michael line $I(M)$ has a $\sigma$-discrete E-net, but does not have a $\sigma$-closure-preserving LE-net.

(2) The same is true for the Sorgenfrey line $R(S)$.

(3) $\left[0, \omega_{1}\right]$ does not admit a o-closure-preserving E-net.

(4) The quotient space $I(M) / Q$ obtained by identifying the rational points in $I(M)$ does not admit a $\sigma$-closure-preserving $E$-net. In particular the property of having $a$ $\sigma$-closure-preserving E-net is not preserved under closed maps.

Proof. (1) and (2) (simultaneously). Let $\mathscr{F}$ be a $\sigma$-discrete net of closed sets in the unit interval $I$ (resp. the real line $R$ ) with the usual topology. It is easy to see that $\mathscr{F}$ is a $\sigma$-discrete $E$-net on $I(M)(\operatorname{resp} . R(S))$. But $I(M)(\operatorname{resp} . R(S))$ does not admit a $\sigma$-closure-preserving $L E$-net because it is not a $\sigma$-space.

(3) For any $\sigma$-closure-preserving collection $\mathscr{F}$ of $\left[0, \omega_{1}\right], \mathcal{F}$ fails to be an $E$-net at $\omega_{1}$; indeed, $\operatorname{Cl}\left(\left\{\bar{F}: F \in \mathscr{F}, \omega_{1} \notin \bar{F}\right\}^{*}\right) \cap\left\{\omega_{1}\right\}=\varnothing$.

(4) If $I(M) / Q$ had a $\sigma$-closure-preserving $E$-net, then every point in $I(M) / Q$, in particular the quotient image of $Q$, would be a $G_{\delta}$-set of $I(M) / Q$; but this is impossible because $Q$ is not a $G_{\delta}$-set of $I(M)$.

\section{Characterizations of $E M_{3}$.}

Lemma 3.1 [ $\mathbf{O}_{2}$, Lemma 3.1]. Let $X$ be a submetrizable space (that is, $X$ admits a weaker metric topology), and let $थ$ be a $\sigma$-discrete collection of cozero sets of $X$. Then there exist a metric space $M$ and a one-to-one map $f: X \rightarrow M$ such that $f(U)$ is an open set of $M$ for every $U \in \mathcal{Q}$.

The following lemma plays a fundamental role in this paper.

LEMMA 3.2. Let $X$ be a paracompact $\sigma$-space and let $\mathscr{F}=\cup_{i=1}^{\infty} \mathscr{F}_{i}$ be a collection of closed sets of $X$ such that $\mathscr{F}_{i}$ is closure-preserving for each $i$. Then there exist a metric space $M$ and a one-to-one map $f: X \rightarrow M$ such that $f(F)$ is a closed set of $M$ for every $F \in \mathscr{F}$ and such that $f\left(\mathscr{F}_{i}\right)$ is closure-preserving in $M$ for every $i$.

Proof. Let $\mathscr{B}=\cup_{i=1}^{\infty} \mathscr{R}_{i}$ be a net of $X$ consisting of closed sets such that $\mathscr{B}_{i}$ is discrete for each $i$. For each $i$ let $\mathscr{V}_{i}=\left\{V_{i}(B): B \in \mathscr{B}_{i}\right\}$ be a discrete collection of open sets of $X$ such that $B \subset V_{i}(B)$ for each $B \in \mathscr{B}_{i}$. For $i, j=1,2, \ldots, B \in \mathscr{B}_{i}$, put

$$
W_{i}^{j}(B)=V_{i}(B) \cap\left(X-\left\{F: F \in \mathscr{F}_{j}, F \cap B=\varnothing\right\}^{*}\right) .
$$

Then $W_{i}^{j}(B)$ is an open set of $X$, and $\left\{W_{i}^{j}(B): B \in \mathscr{B}_{i}\right\}$ is discrete in $X$. Hence Lemma 3.1 applies to give a metric space $M$ and a one-to-one map $f: X \rightarrow M$ such that $f\left(W_{i}^{j}(B)\right)$ is an open set of $M$ for every $B \in \mathscr{B}_{i}, i, j=1,2, \ldots$ It is then obvious that for each $i, f\left(\mathscr{F}_{i}\right)$ is a closure-preserving collection of closed sets of $M$. This completes the proof.

Definition 3.3. Let $X \in E M_{3}$ and let $\{\mathscr{F}, \mathcal{V}, \mathcal{E}, \mathcal{\delta}\}$ be a quartet of collections of subsets of $X$. The quartet is called an E-quartet if we can write $\mathscr{F}=\cup_{i=1}^{\infty} \mathscr{F}_{i}$, $\mathcal{V}=\cup_{i=1}^{\infty} \mathcal{V}_{i}, \mathcal{E}=\cup_{i=1}^{\infty} \mathcal{E}_{i}, \delta=\cup_{i=1}^{\infty} \mathcal{S}_{i}$ and if the following four conditions are satisfied:

$\left(1_{q}\right) \mathscr{F}$ is a net on $X$ consisting of closed sets. 
$\left(2_{\mathrm{q}}\right)$ For each $i, \mathfrak{V}_{i}$ is a discrete collection of open sets of $X$ written as $\mathcal{V}_{i}=\left\{V_{i}(F)\right.$ : $F \in \mathscr{F}_{i}$ \} in such a manner that $F \subset V_{i}(F)$ for each $F \in \mathscr{F}_{i}$.

$\left(3_{\mathrm{q}}\right) \mathcal{E}$ is an E-net on $X$ consisting of closed sets and $\mathcal{E}_{i}$ is closure-preserving for each $i$.

$\left(4_{q}\right) \mathcal{S}$ is a quasi-base $e^{4}$ for $X$ consisting of closed sets and $\mathcal{S}_{i}$ is closure-preserving for each $i$.

By Heath $[\mathbf{H}]$, Gruenhage $[\mathbf{G}]$ and Junnila $[\mathbf{J}]$, each member of $E M_{3}$ admits an $E$-quartet.

Definition 3.4. Let $X$ be a member of $E M_{3}$ with an $E$-quartet $\{\mathscr{F}, \mathcal{T}, \mathcal{E}, \mathcal{\delta}\}$. A map $f: X \rightarrow Y$ onto a normal space $Y$ is called an $E$-map with respect to the $E$-quartet if the following five conditions are satisfied:

$\left(0_{\mathrm{f}}\right) f$ is one-to-one.

$\left(1_{\mathrm{f}}\right) f(F)$ is a closed set for every $F \in \mathscr{F}$.

$\left(2_{\mathrm{f}}\right) f(V)$ is an open set for every $V \in \mathcal{V}$.

$\left(3_{\mathrm{f}}\right) f(E)$ is a closed set for every $E \in \mathcal{E}$, and $f\left(\mathcal{E}_{i}\right)$ is closure-preserving in $Y$ for every $i$.

$\left(4_{\mathrm{f}}\right) f(S)$ is a closed set for every $S \in \mathcal{S}$, and $f\left(\mathcal{S}_{i}\right)$ is closure-preserving in $Y$ for every $i$.

Noting that $\{X-V: V \in \mathscr{V}\}$ is a $\sigma$-closure-preserving collection of closed sets of $X$, we have the following result by virtue of Lemma 3.2.

Proposition 3.5. Let $X$ be a member of $E M_{3}$. Then for any $E$-quartet of $X$ there exist a metric space $M$ and an E-map $f: X \rightarrow M$ with respect to the E-quartet.

The following lemma is well known (see, for example, [E, 2.3.16]).

Lemma 3.6. Let $X$ be a space and let $C, K$ be disjoint closed sets of $X$. Let थ be a countable open cover of $X$ such that for each $U \in \mathcal{Q}$, either $\bar{U} \cap C=\varnothing$ or $\bar{U} \cap K=\varnothing$. Then $C$ and $K$ are separated by a closed set $S$ such that $S \subset\{\operatorname{Bd} U: U \in \mathcal{Q}\}^{*}$.

Now we have the following result frequently used later.

Proposition 3.7. Let $X$ be a member of $E M_{3}$ with an E-quartet $\{\mathcal{F}, \mathcal{V}, \mathcal{E}, \mathcal{S}\}$. Let $f$ : $X \rightarrow Y$ be an E-map with respect to the E-quartet onto a normal space $Y$. Then Ind $X \leqslant$ Ind $Y$.

Proof. The proof is by induction on Ind $Y$. If $Y=\varnothing$ then the proposition is trivial. Suppose that the proposition is valid when Ind $Y \leqslant n-1$ and consider the case of Ind $Y=n$. To show Ind $X \leqslant n$, let $C, K$ be disjoint closed sets of $X$. For the time being, fix a point $x$ in $X-C$ arbitrarily. We show that there exists an open neighborhood $W$ of $x$ such that $\bar{W} \cap C=\varnothing$ and Ind $\mathrm{Bd} W \leqslant n-1$. Let $\mathcal{E}(x)$ be a subcollection of $\mathcal{E}$ such that $x \in X-\mathcal{E}(x)^{*} \subset X-C$ and $\mathcal{E}(x)^{*}$ is a closed set. Write $\mathcal{E}(x)=\cup_{i=1}^{\infty} \mathcal{E}_{i}(x)$ where $\mathcal{E}_{i}(x) \subset \mathcal{E}_{i}$. Put $\delta_{i}(x)=\left\{S \in \delta_{i}: S \cap \mathcal{E}(x)^{*}=\varnothing\right\}$

\footnotetext{
${ }^{4}$ A collection $\mathcal{S}$ of subsets of a space $X$ is called a quasi-base for $X$ if for any point $x$ and any open neighborhood $U$ of $x$ there exists a member $S$ of $\mathcal{S}$ such that $x \in \operatorname{Int} S \subset S \subset U$.
} 
and $\delta(x)=\cup_{i=1}^{\infty} \delta_{i}(x)$. Fix $i_{0}$ so that $x \in \operatorname{Int} \delta_{i_{0}}(x)^{*}$. By $\left(3_{\mathrm{f}}\right)$ and $\left(4_{\mathrm{f}}\right)$ there exist open sets $O_{j}, j=1,2, \ldots$, of $Y$ such that

$$
f\left(\bigcup_{i=1}^{j} \varsigma_{i}(x)^{*}\right) \cup f\left(\delta_{i_{0}}(x)^{*}\right) \subset O_{j} \subset \overline{O_{j}} \subset Y-f\left(\delta_{j}(x)^{*}\right)
$$

and

$$
\text { Ind } \mathrm{Bd} O_{j} \leqslant n-1 .
$$

Define $W=\bigcap_{j=1}^{\infty} f^{-1}\left(O_{j}\right)$. Then

$$
x \in W \subset \bar{W} \subset \bigcap_{j=1}^{\infty} f^{-1}\left(\bar{O}_{j}\right) \subset X-\varepsilon(x)^{*} \subset X-C .
$$

To show that $W$ is open, let $x^{\prime} \in W$. Since $x^{\prime} \in X-\mathcal{E}(x)^{*}$ and $\varepsilon(x)^{*}$ is a closed set, it follows from $\left(4_{\mathrm{q}}\right)$ that $x^{\prime} \in \operatorname{Int} \delta_{m}(x)^{*}$ for some $m$. Then

$$
x^{\prime} \in \bigcap_{j=1}^{m-1} f^{-1}\left(O_{j}\right) \cap \operatorname{Int} \delta_{m}(x)^{*} \subset W,
$$

which implies that $W$ is open. To show Ind $\mathrm{Bd} W \leqslant n-1$, note that, for any subset $Z$ of $X, f \mid Z: Z \rightarrow f(Z)$ is again an $E$-map with respect to the $E$-quartet $\{\mathscr{F} \mid Z$, $\mathfrak{V}|Z, \mathcal{E}| Z, \mathcal{S} \mid Z\}$ on $Z$. Hence we may apply induction hypothesis to obtain Ind $f^{-1}\left(\operatorname{Bd} O_{j}\right) \leqslant n-1, j=1,2, \ldots$, which yields

$$
\text { Ind Bd } \begin{aligned}
W & \leqslant \operatorname{Ind}\left(\bigcup_{j=1}^{\infty} \operatorname{Bd} f^{-1}\left(O_{j}\right)\right) \\
& =\max \left\{\operatorname{Ind} \operatorname{Bd} f^{-1}\left(O_{j}\right): j=1,2, \ldots\right\} \\
& \leqslant \max \left\{\text { Ind } f^{-1}\left(\operatorname{Bd} O_{j}\right): j=1,2, \ldots\right\} \leqslant n-1 .
\end{aligned}
$$

Hence $W$ is a required open neighborhood of $x$; we have thus finished "local" separation.

Now put

$$
\begin{aligned}
& \mathscr{F}_{i}(C)=\left\{F \in \mathscr{F}_{i}: F \subset W \text { for some open set } W\right. \text { with } \\
& \qquad \bar{W} \cap C=\varnothing \text { and Ind Bd } W \leqslant n-1\} .
\end{aligned}
$$

Then by $\left(1_{\mathrm{q}}\right)$ and by the "local" separation above, we have $\cup_{i=1}^{\infty} \mathscr{F}_{i}(C)^{*}=X-C$. For each $F \in \mathscr{F}_{i}(C)$, fix such a $W$ and denote it by $W_{i}(C, F)$. On the other hand, by $\left(1_{\mathrm{f}}\right)$ and $\left(2_{\mathrm{f}}\right)$, there exist open sets $H_{i}(F), F \in \mathscr{F}_{i}$, of $Y$ such that $f(F) \subset H_{i}(F) \subset$ $\mathrm{Cl} H_{i}(F) \subset f\left(V_{i}(F)\right.$ ) and Ind $\mathrm{Bd} H_{i}(F) \leqslant n-1$ (where the set $V_{i}(F)$ is as in Definition 3.3(2 $)$ ). By induction hypothesis again,

$$
\text { Ind } \mathrm{Bd} f^{-1}\left(H_{i}(F)\right) \leqslant \operatorname{Ind} f^{-1}\left(\mathrm{Bd} H_{i}(F)\right) \leqslant n-1 \text {. }
$$

Put for each $F \in \mathscr{F}_{i}(C)$,

$$
D_{i}(C, F)=W_{i}(C, F) \cap f^{-1}\left(H_{i}(F)\right)
$$

Then

Ind Bd $D_{i}(C, F) \leqslant \max \left\{\right.$ Ind Bd $W_{i}(C, F)$, Ind Bd $\left.f^{-1}\left(H_{i}(F)\right)\right\} \leqslant n-1$. 
Put $D_{i}(C)=\left\{D_{i}(C, F): F \in \mathscr{F}_{i}(C)\right\}^{*}$. Since $D_{i}(C, F) \subset V_{i}(F),\left(2_{\mathrm{q}}\right)$ implies that $\left\{D_{i}(C, F): F \in \mathscr{F}_{i}(C)\right\}$ is discrete. Thus Ind $\mathrm{Bd} D_{i}(C) \leqslant n-1, i=1,2, \ldots$ By the same discreteness and by the fact $D_{i}(C, F) \subset W_{i}(C, F) \subset \mathrm{Cl} W_{i}(C, F) \subset X-C$, we have $C \cap \mathrm{Cl} D_{i}(C)=\varnothing$ for every $i=1,2, \ldots$ We also obtain $\cup_{i=1}^{\infty} D_{i}(C)=X$ - $C$ because $\cup_{i=1}^{\infty} \mathscr{F}_{i}(C)^{*}=X-C$.

Quite similarly we can obtain open subsets $D_{i}(K), i=1,2, \ldots$, such that Ind $\mathrm{Bd} D_{i}(K) \leqslant n-1, K \cap \mathrm{Cl} D_{i}(K)=\varnothing$ and $\cup_{i=1}^{\infty} D_{i}(K)=X-K$. Hence, applying Lemma 3.6, we have a closed set $B$ separating $C$ and $K$ such that

$$
B \subset\left(\bigcup_{i=1}^{\infty} \operatorname{Bd} D_{i}(C)\right) \cup\left(\bigcup_{i=1}^{\infty} \operatorname{Bd} D_{i}(K)\right) .
$$

By the countable sum theorem for Ind, we have Ind $B \leqslant n-1$. Thus Ind $X \leqslant n$, which completes the proof of Proposition 3.7.

We can now prove a characterization theorem for $E M_{3}$.

THEOREM 3.8. The following statements about a space $X$ are equivalent:

(1) $X$ is a stratifiable space with a $\sigma$-closure-preserving $E$-net.

(2) $X$ is the perfect image of a stratifiable space $X_{0}$ with $\operatorname{dim} X_{0} \leqslant 0$.

(3) $X$ is the closed image of a stratifiable space $X_{0}$ with ind $X_{0} \leqslant 0$.

(4) $X$ is a stratifiable space with a $\sigma$-closure-preserving LE-net.

Proof. The implications (2) $\rightarrow(3)$ and (4) $\rightarrow$ (1) are obvious. To show (1) $\rightarrow$ (2) let $X$ be a member of $E M_{3}$ with an $E$-quartet $\{\mathscr{F}, \mathcal{T}, \mathcal{E}, \mathcal{S}\}$. By Proposition 3.5 there exists an $E$-map $f: X \rightarrow M$ onto a metric space $M$ with respect to $\{\mathscr{F}, \mathcal{V}, \mathcal{E}, \mathcal{\delta}\}$. By Morita [M], $M$ is the image of a metric space $P$ with $\operatorname{dim} P \leqslant 0$ under a perfect map $g$. Now let $T$ be the fiber product of $P$ and $X$ with respect to $g$ and $f$, that is,

$$
T=\{(p, x) \in P \times X: g(p)=f(x)\}
$$

with the topology induced from $P \times X$. Let $t_{P}, t_{X}$ be the restrictions to $T$ of the projections from $P \times X$ onto $P$ and $X$, respectively. We thus have the following commutative diagram:

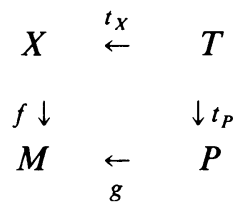

It is a well-known property of fiber products that the perfectness of $g$ implies the perfectness of $t_{X}$ (see [Pe, Lemma 7.5.13]). $T$ is stratifiable by [C, Theorems 2.3, 2.4]. Hence what should be proved is the zero-dimensionality of $T$. By Proposition 2.2, $P$ admits an $E$-quartet $\left\{\mathscr{F}_{P}, \mathscr{V}_{P}, \mathcal{E}_{P}, \mathcal{S}_{P}\right\}$. Now define

$$
\begin{aligned}
& \mathscr{F}_{T}=\left\{t_{P}^{-1}\left(F_{p}\right) \cap t_{X}^{-1}(F): F_{P} \in \mathscr{F}_{P}, F \in \mathscr{F}\right\}, \\
& \mathscr{V}_{T}=\left\{t_{P}^{-1}\left(V_{P}\right) \cap t_{X}^{-1}(V): V_{P} \in \mathscr{V}_{P}, V \in \mathscr{V}\right\}, \\
& \mathcal{S}_{T}=\left\{t_{P}^{-1}\left(S_{P}\right) \cap t_{X}^{-1}(S): S_{P} \in \mathcal{S}_{P}, S \in \mathcal{S}\right\}, \text { and } \\
& \mathcal{E}_{T}=\left\{t_{P}^{-1}\left(E_{P}\right): E_{P} \in \mathcal{E}_{P}\right\} \cup\left\{t_{X}^{-1}(E): E \in \mathcal{E}\right\} .
\end{aligned}
$$


Then it is easy to see that the quartet $\left\{\mathscr{F}_{T}, \mathcal{V}_{T}, \mathcal{E}_{T}, \mathcal{S}_{T}\right\}$ is an $E$-quartet of $T$. Furthermore, the map $t_{P}$ is an $E$-map with respect to $\left\{\mathscr{F}_{T}, \mathscr{V}_{T}, \mathcal{E}_{T}, \mathcal{S}_{T}\right\}$ because, in general, $t_{P}\left(t_{P}^{-1}\left(P^{\prime}\right) \cap t_{X}^{-1}\left(X^{\prime}\right)\right)=P^{\prime} \cap g^{-1} \circ f\left(X^{\prime}\right)$ for any $P^{\prime} \subset P$ and $X^{\prime} \subset X$, and because $f$ is an $E$-map with respect to $\{\mathscr{F}, \mathcal{V}, \mathcal{E}, \mathcal{\delta}\}$. Hence, applying Proposition 3.7, we have Ind $T \leqslant 0$. Thus the implication (1) $\rightarrow(2)$ has been proved.

To show (3) $\rightarrow(4)$ let $X_{0}$ be a stratifiable space with ind $X_{0} \leqslant 0$ and let $f: X_{0} \rightarrow X$ be a closed map. Note that every net on $X_{0}$ is an $E$-net; hence $X_{0}$ is a member of $E M_{3}$ by Heath [H]. It now follows from the implication (1) $\rightarrow(2)$ that $X_{0}$ is the image of a stratifiable space $X_{1}$ with $\operatorname{dim} X_{1} \leqslant 0$ under a perfect map $h$. Since every net on $X_{1}$ is an $L E$-net, it follows from Heath [H] again that $X_{1}$ admits a $\sigma$-closure-preserving $L E$-net. Hence, applying Proposition 2.3 to the closed map $f \circ h$, we see that $X$ admits a $\sigma$-closure-preserving $L E$-net. On the other hand $X$ is stratifiable by Borges [B, Theorem 3.1]. This completes the proof of Theorem 3.8.

COROLlaRy 3.9. The class $E M_{3}$ is countably productive, hereditary and preserved under closed maps.

Proof. This is immediate from Theorem 3.8, Proposition 2.6 and the analogous result for $M_{3}$ due to Ceder [C] and Borges [B].

A topological class $\mathcal{C}$ is called perfect $\left(\mathrm{Nagami}\left[\mathbf{N}_{\mathbf{1}}\right]\right.$, also see $\left.\left[\mathbf{N}_{2}\right]\right)$ if it is countably productive, hereditary, preserved under perfect maps, included in the class of normal spaces, and every member of $\mathcal{C}$ is the perfect image of a zero-dimensional (in the sense of dim) member of $\mathcal{C}$. Theorem 3.8 and Corollary 3.9 say

COROLlaRY 3.10. The class $E M_{3}$ is the maximal perfect subclass of $M_{3}$.

Recently Itō [I] has presented a free $L$-space, a certain closed image of which is not a free $L$-space. But we have

COROLlARY 3.11. Every closed image of a free L-space is a member of $E M_{3}$.

Proof. By Nagami $\left[\mathbf{N}_{4}\right.$, Theorem 2.10] and Theorem 3.8, every free $L$-space is a member of $E M_{3}$ (it is also easy to directly prove that every free $L$-space admits a $\sigma$-closure-preserving $E$-net). Hence this corollary is immediate from Corollary 3.9.

4. Dimension for $E M_{3}$. We begin with the equidimensional $G_{\delta}$-envelope theorem. To show this, the following lemma is useful.

LEMMA 4.1 (OKA [O $\mathbf{O}_{4}$, LEMMA 3.3]). Let $X$ be a hereditarily normal space and let $f$ : $X \rightarrow L$ be a map onto a metric space $L$. Then for any subset $Y \subset X$, there exist a $G_{\delta}$-set $Z$ of $X$, a metric space $M$ and maps $g: Z \rightarrow M, h: M \rightarrow f(Z)$ such that

(i) $Y \subset Z$,

(ii) $\operatorname{dim} g(Y) \leqslant \operatorname{dim} Y$ and

(iii) $f \mid Z=h \circ g$.

TheOREM 4.2. Let $X \in E M_{3}$ and let $Y$ be a subset of $X$ with $\operatorname{dim} Y \leqslant n$. Then there exists $a G_{\delta}$-set $G$ of $X$ such that $Y \subset G$ and $\operatorname{dim} G \leqslant n$.

Proof. Let $f: X \rightarrow L$ be an $E$-map onto a metric space $L$ with respect to an $E$-quartet, say $\{\mathscr{F}, \mathcal{T}, \mathcal{E}, \delta\}$, on $X$. By the above lemma there exist a $G_{\delta}$-set $Z$ of $X$, a 
metric space $M$ and maps $g: Z \rightarrow M, h: M \rightarrow f(Z)$ satisfying (i), (ii), (iii) above. Since $\operatorname{dim} g(Y) \leqslant n$ and $M$ is metrizable, we can find a $G_{\delta}$-set $H$ of $M$ such that $g(Y) \subset H$ and $\operatorname{dim} H \leqslant n$ (see, for example, [E, 4.1.19]). Define $G=g^{-1}(H)$. Then $G$ is a $G_{\delta}$-set of $Z$, and hence of $X$. To show $\operatorname{dim} G \leqslant n$, note that $g \mid G$ is an $E$ map with respect to $\{\mathscr{F}|G, \mathcal{V}| G, \mathcal{E}|G, \mathcal{S}| G\}$ because $f \mid G$ is so and because $f|G=h \circ g| G$ by (iii). Hence by Proposition 3.7 we have Ind $G \leqslant$ Ind $H$. Consequently

$$
\operatorname{dim} G \leqslant \operatorname{Ind} G \leqslant \operatorname{Ind} H=\operatorname{dim} H \leqslant n,
$$

as required. This completes the proof.

The following theorem occupies the central position in dimension theory of $E M_{3}$. The key argument of the proof has already appeared in the proof of Theorem 3.8.

THEOREM 4.3. The following statements about a space $X$ are equivalent:

(1) $X \in E M_{3}$ and $\operatorname{dim} X \leqslant n$.

(2) $X$ is the image of a stratifiable space $X_{0}$ with $\operatorname{dim} X_{0} \leqslant 0$ under a perfect map of order not greater than $n+1$.

(3) $X$ is a stratifiable space which is the union of $G_{\delta}$-sets $X_{i}, 1 \leqslant i \leqslant n+1$, with $\operatorname{dim} X_{i} \leqslant 0$.

(4) $X \in E M_{3}$ and Ind $X \leqslant n$.

Proof. (1) $\rightarrow(2)$. Let $X$ be a member of $E M_{3}$ such that $\operatorname{dim} X \leqslant n$. Let $\{\mathscr{F}, \mathcal{V}, \mathcal{E}, \mathcal{S}\}$ be an $E$-quartet of $X$. By Proposition 3.5 there exist a metric space $L$ and an $E$-map $f: X \rightarrow L$ with respect to the $E$-quartet. By Pasynkov's factorization theorem [P, Theorem 29], there exist a metric space $M$ and maps $g: X \rightarrow M, h$ : $M \rightarrow L$ such that $\operatorname{dim} M \leqslant n$ and $f=h \circ g$. It then follows from Morita [M] that $M$ is the image of a metric space $P$ with $\operatorname{dim} P \leqslant 0$ under a perfect map $r$ such that ord $r \leqslant n+1$. Let $T$ be the fiber product of $P$ and $X$ with respect to $r$ and $g$, and let $t_{p}, t_{X}$ be the restrictions to $T$ of the projections from $P \times X$ onto $P$ and $X$, respectively. We thus obtain the following commutative diagram:

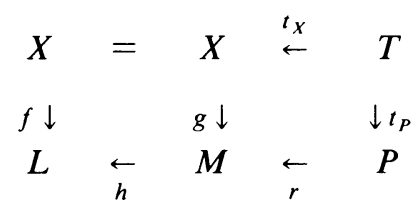

It is obvious that $t_{X}$ is a perfect map of order not greater than $n+1$ and that $T$ is a stratifiable space. Note that $g$ is an $E$-map with respect to $\{\mathscr{F}, \mathcal{V}, \mathcal{E}, \delta\}$ because $f$ is so and $f=h \circ g$. Now, as in the proof of Theorem 3.8, $t_{P}$ is also an $E$-map with respect to a certain $E$-quartet of $T$, and hence $\operatorname{dim} T \leqslant 0$ by Proposition 3.7.

$(2) \rightarrow(3)$. Let $t: X_{0} \rightarrow X$ be a perfect map from a stratifiable space $X_{0}$ with $\operatorname{dim} X_{0} \leqslant 0$ onto a space $X$ such that ord $t \leqslant n+1$. Put $Y_{i}=\left\{x \in X:\left|t^{-1}(x)\right|=i\right\}$, $1 \leqslant i \leqslant n+1$. It then follows from Nagami $\left[\mathbf{N}_{2}\right.$, Lemma 4] that $\operatorname{dim} Y_{i} \leqslant 0$ for each $i=1,2, \ldots, n+1$. Since $X$ is a member of $E M_{3}$ by Theorem 3.8, we may apply Theorem 4.2 to obtain $G_{\delta}$-sets $X_{i}, 1 \leqslant i \leqslant n+1$, such that $\operatorname{dim} X_{i} \leqslant 0$ and $Y_{i} \subset X_{i}$.

The implication $(4) \rightarrow(1)$ is trivial. 
Finally the implication (3) $\rightarrow$ (4) is assured by the following theorem (but the fact Ind $X \leqslant n$ only is direct from (3) as a consequence general for hereditarily normal spaces).

THEOREM 4.4. Let $X$ be a normal $\sigma$-space expressed as the finite union of $G_{\delta}$-sets $X_{i}$, $1 \leqslant i \leqslant k$, such that $\operatorname{dim} X_{i} \leqslant 0$. Then $X$ admits a $\sigma$-closure-preserving LE-net.

Proof. The proof is by induction on $k$. When $k=1$, the theorem is trivial. Now suppose that the theorem is valid when $k=m-1$, and consider the case $k=m$. Put $Y_{m}=X-X_{m}$. Then by induction hypothesis and Lemma 2.5, the normal $\sigma$-space $Y_{m}$ admits a $\sigma$-closure-preserving $L E$-net, say $\mathcal{E}$, consisting of closed sets of $Y_{m}$. Write $Y_{m}=\cup_{i=1}^{\infty} C_{i}$ with closed sets $C_{i}$ such that $C_{i} \subset C_{i+1}$, and put $\varepsilon_{i}=\mathcal{E} \mid C_{i}$. Let $\mathscr{F}$ be a $\sigma$-locally finite net of $X$. Now consider the $\sigma$-closure-preserving collection $\cup_{i=1}^{\infty} \mathcal{E}_{i} \cup \mathcal{F}$ of $X$. To show that the collection is an $L E$-net on $X$, let $C, K$ be disjoint closed sets of $X$. Since $X$ is hereditarily normal and Ind $X_{m} \leqslant 0$, there exists a closed set $S$ separating $C$ and $K$ such that $S \cap X_{m}=\varnothing$. Represent $X$ as the disjoint union $V \cup S \cup W$, where $V$ and $W$ are open sets of $X$ including $C$ and $K$ respectively. Write $V=\cup_{i=1}^{\infty} V_{i}$ with open sets $V_{i}$ such that $\bar{V}_{i} \subset V_{i+1}$ for every $i$. For each $i$ take a subcollection $\mathscr{F}_{i}$ of $\mathcal{E}_{i}$ such that

$$
(W \cup S) \cap C_{i} \subset \mathscr{F}_{i}^{*} \subset C_{i}-\left(\bar{V}_{i} \cup C\right)
$$

and $\mathcal{F}_{i}^{*}$ is a closed set of $C_{i}$. Now put

$$
B=W \cup\left(\bigcup_{i=1}^{\infty} \mathscr{F}_{i}^{*}\right) .
$$

It is easy to see that $B$ is a closed set of $X$ including $K$ and not meeting $C$. Since $W$ is the union of some members of $\mathscr{F}, B$ is the union of some members of $\cup_{i=1}^{\infty} \mathcal{E}_{i} \cup \mathcal{F}$. Thus $\cup_{i=1}^{\infty} \mathcal{E}_{i} \cup \mathcal{F}$ is a $\sigma$-closure-preserving $L E$-net on $X$. This completes the proof of Theorem 4.4 and, therefore, of Theorem 4.3.

REMARK. Slightly modifying the above proof, we can weaken the condition " $X_{i}$ is $G_{\delta}$ " in Theorem 4.4 to " $X_{i}$ is either $G_{\delta}$ or $F_{\sigma}$ ".

As a trivial version of Theorem 4.4, we have the following result which tells us that the dimension theory does not work well in the remainder $M_{3}-E M_{3}$.

COROllaRY 4.5. Let $X$ be a normal $\sigma$-space not admitting a $\sigma$-closure-preserving LE-net. Then either

(1) X cannot be decomposed into finitely many zero-dimensional (in the sense of dim) subsets, or

(2) there exists a zero-dimensional (in the sense of $\operatorname{dim}$ ) subset of $X$ not admitting an equidimensional $G_{\delta}$-envelope.

As an immediate consequence of Theorem 4.3, we have

Corollary 4.6. Let $X$ be a stratifiable space with ind $X \leqslant 0$. Then $\operatorname{dim} X=\operatorname{Ind} X$.

REMARK. This result, however, is generalized to paracompact $\sigma$-spaces in my recent paper $\left[\mathbf{O}_{5}\right]$. 
We conclude this section with the following result, an immediate consequence of Corollary 3.11 and Theorem 4.3.

Corollary 4.7. Let $X$ be the closed image of a free $L$-space. Then $\operatorname{dim} X=\operatorname{Ind} X$.

5. Other spaces admitting $\sigma$-closure-preserving $E$-nets. Let $\bigodot$ be a topological property. A space is called peripherally $\mathcal{C}$ if every point in the space admits an open neighborhood base, the boundary of each member of which is $\mathcal{C}$.

THEOREM 5.1. (1) A peripherally $\sigma$-discrete, paracompact $\sigma$-space admits a $\sigma$ closure-preserving E-net.

(2) A peripherally $\sigma$-compact, stratifiable space admits a $\sigma$-closure-preserving E-net.

Proof. We shall prove (1) and (2) simultaneously. Let $\mathscr{F}$ be a $\sigma$-locally finite net (resp. a $\sigma$-closure-preserving quasi-base) of $X$ consisting of closed sets. To show that $\mathscr{F}$ itself is an $E$-net on $X$ let $x$ be a point of $X$ and $V$ an open neighborhood of $x$. Take an open set $U$ such that $x \in U \subset \bar{U} \subset V$ and $\mathrm{Bd} U$ is $\sigma$-discrete (resp. $\sigma$-compact). Write $U=\cup_{i=1}^{\infty} U_{i}$ with open sets $U_{i}$ such that $\bar{U}_{i} \subset U_{i+1}$ for every $i$. Write $\operatorname{Bd} U=\cup_{i=1}^{\infty} C_{i}$ with discrete (resp. compact) closed sets $C_{i}, i=1,2, \ldots$ There exists, for each $i$, a discrete (resp. finite) subcollection $\mathscr{F}_{i}$ of $\mathscr{F}$ such that $C_{i} \subset \mathscr{F}_{i}^{*} \subset X$ $-\left(\bar{U}_{i} \cup\{x\}\right)$. Then $\cup_{i=1}^{\infty} \mathscr{F}_{i}^{*} \cup(X-\bar{U})$ is a closed set of $X$ including $X-V$, not meeting $\{x\}$ and expressed as a union of members of $\mathscr{F}$. Thus $\mathscr{F}$ is an $E$-net of $X$, which completes the proof.

Now we have the following generalization of Corollary 4.6.

COROLlaRY 5.2. Let $X$ be a peripherally $\sigma$-compact (or peripherally $\sigma$-discrete) stratifiable space. Then $\operatorname{dim} X=\operatorname{Ind} X$.

We next verify a countable sum theorem for $\sigma$-closure-preserving $L E$-nets.

THEOREM 5.3. Let $X$ be a normal space expressed as the countable union of closed sets $X_{i}, i=1,2, \ldots$, each of which admits a $\sigma$-closure-preserving LE-net. Then X has a o-closure-preserving LE-net.

Proof. Note that $X$ is perfectly normal because each $X_{i}$ is. Let $\mathcal{E}_{i}$ be a $\sigma$-closurepreserving $L E$-net of $X_{i}$. It is clear that $\cup_{i=1}^{\infty} \mathcal{E}_{i}$ is $\sigma$-closure-preserving in $X$. To show that $\cup_{i=1}^{\infty} \mathcal{E}_{i}$ is an $L E$-net, let $C$ and $K$ be disjoint closed sets of $X$. Write $X-C=\cup_{i=1}^{\infty} V_{i}$ with open sets $V_{i}$ such that $\bar{V}_{i} \subset V_{i+1}$. For each $i$ let $\mathscr{F}_{i}$ be a subcollection of $\mathcal{E}_{i}$ such that $\mathscr{F}_{i}^{*}$ is a closed set of $X_{i}$ and $C \cap X_{i} \subset \mathscr{F}_{i}^{*} \subset X_{i}-(K \cup$ $\left.\bar{V}_{i}\right)$. It is then obvious that $\cup_{i=1}^{\infty} \mathscr{F}_{i}^{*}$ is a closed set of $X$ and $C \subset \cup_{i=1}^{\infty} \mathscr{F}_{i}^{*} \subset X-K$. This completes the proof.

The following result is immediate from Theorem 5.3, Proposition 2.2 and Ceder [C, Theorem 8.3].

Corollary 5.4. A chunk complex (and hence a $C W$-complex) is a member of $E M_{3}$.

We list several unsolved problems below. 
Problem 5.5. (1) Does every stratifiable space admit a $\sigma$-closure-preserving E-net? By virtue of Theorem 3.8, this is equivalent to:

(2) (Nagami [ $\mathbf{N}_{1}$, Problem 4]) Is every stratifiable space a perfect image of a zero-dimensional (in the sense of $\mathrm{dim}$ ) stratifiable space?

The author also does not know whether the inclusion $E M_{3} \subset M_{1}$ (or $M_{1} \subset E M_{3}$ ) holds or not.

Problem 5.6. Let $X$ be a paracompact $\sigma$-space admitting a $\sigma$-closure-preserving E-net. Then:

(1) Does the equality $\operatorname{dim} X=$ Ind $X$ hold?

(2) Is $X$ a perfect image of a zero-dimensional (in the sense of dim) paracompact $\sigma$-space? More weakly:

(3) Does $X$ admit a $\sigma$-closure-preserving LE-net?

In the specific case of ind $X \leqslant 0$, (1) admits an affirmative answer by the inequality Ind $X \leqslant \operatorname{dim} X+$ ind $X$ for every nonempty paracompact $\sigma$-space $X$ $\left[\mathbf{O}_{\mathbf{5}}\right]$; (2) is also affirmative, that is, a paracompact $\sigma$-space of ind $\leqslant 0$ is the perfect image of a paracompact $\sigma$-space of $\operatorname{dim} \leqslant 0$.

To outline the proof, let $X$ be a nonempty paracompact $\sigma$-space with ind $X=0$. Let $\mathscr{F}=\cup_{i=1}^{\infty} \mathscr{F}_{i}$ and $\mathfrak{V}=\cup_{i=1}^{\infty} \mathcal{V}_{i}$ be as in Definition 3.3. Let $f: X \rightarrow M$ be a one-to-one map onto a metric space $M$ such that $f\left(\mathscr{V}_{i}^{*}\right)$ is open and $f\left(\mathscr{F}_{i}^{*}\right)$ is closed for every $i$. In $\left[\mathrm{O}_{5}\right.$, Lemma 5] it is proved that, in general, Ind $X \leqslant$ Ind $M+$ ind $X$ for any such map $f: X \rightarrow M$. The metric space $M$ is the image of a metric space $L$ with $\operatorname{dim} L=0$ under a perfect map $g$. Let $T$ be the fiber product of $L$ and $X$ with respect to $g$ and $f$. Let $t_{L}, t_{X}$ be the restrictions to $T$ of the projections from $L \times X$ onto $L$ and $X$, respectively. Then, since the map $t_{L}$ is of the "same type" as $f$, we have Ind $T \leqslant \operatorname{Ind} L+$ ind $T=$ ind $T$. But, in the present case, ind $T \leqslant \operatorname{ind}(L \times X)$ $=0$; hence Ind $T=0$. It is clear that $T$ is a paracompact $\sigma$-space and $t_{X}$ is a perfect map. This completes the proof.

Problem 5.7. Let $X$ be a stratifiable space expressed as the union of countably many metrizable $\left(G_{\delta^{-}}\right)$subsets. Does the equality $\operatorname{dim} X=$ Ind $X$ hold? More strongly, does $X$ admit a $\sigma$-closure-preserving $E$-net? (A space of this type is a natural generalization of a Lašnev space in view of Lašnev's well-known decomposition theorem [La].)

\section{BIBLIOGRAPHY}

[B] C. R. Borges, On stratifiable spaces, Pacific J. Math. 17 (1966), 1-16.

[C] J. G. Ceder, Some generalizations of metric spaces, Pacific J. Math. 11 (1961), 105-125.

[Cr] G. D. Creede, Concerning semi-stratifiable spaces, Pacific J. Math. 32 (1970), 47-54.

[E] R. Engelking, Dimension theory, North-Holland, Amsterdam, 1978.

[G] G. Gruenhage, Stratifiable spaces are $M_{2}$, Topology Proc. 1 (1976), 221-226.

[H] R. W. Heath, Stratifiable spaces are o-spaces, Notices Amer. Math. Soc. 16 (1969), 761.

[I] M. Itō, Weak L-spaces are free L-spaces, J. Math. Soc. Japan 34 (1982), 507-514.

[J] H. Junnila, Neighbornets, Pacific J. Math. 76 (1978), 83-108.

[La] N. S. Lašnev, Continuous decompositions and closed mappings of metric spaces, Soviet Math. Dokl. 6 (1965), 1504-1506.

$\left[\mathbf{L}_{1}\right]$ I. M. Leibo, On the equality of dimensions for closed images of metric spaces, Soviet Math. Dokl. 15 (1974), 835-839.

$\left[\mathbf{L}_{2}\right] \ldots$, On closed images of metric spaces, Soviet Math. Dokl. 16 (1975), 1292-1295.

[M] K. Morita, A condition for the metrizability of topological spaces and for $n$-dimensionality, Sci. Rep. Tokyo Kyoiku Daigaku Sect. A 5 (1955), 33-36. 
$\left[\mathbf{N}_{\mathbf{1}}\right]$ K. Nagami, Normality of products, Actes Congrès Intern. Math. 2 (1970), 33-37.

$\left[\mathrm{N}_{2}\right] \ldots$, Perfect class of spaces, Proc. Japan Acad. 48 (1972), 21-24.

$\left[\mathrm{N}_{3}\right]$ _. The equality of dimensions, Fund. Math. 106 (1980), 239-246.

$\left[\mathrm{N}_{4}\right] \ldots$ Dimension of free L-spaces, Fund. Math. 108 (1980), 211-224.

$\left[\mathbf{O}_{1}\right]$ S. Oka, A note on the covering dimension of Lašnev spaces, Proc. Japan Acad. Ser. A 54 (1978), 73-75.

$\left[\mathrm{O}_{2}\right] \ldots$, Dimension of finite unions of metric spaces, Math. Japon. 24 (1979), 351-362.

$\left[\mathrm{O}_{3}\right] \ldots$, Every Lašnev space is the perfect image of a zero-dimensional one, Bull. Acad. Polon. Sci. Ser. Sci. Math. 28 (1980), 591-594.

$\left[\mathbf{O}_{4}\right] \ldots$ Free patched spaces and fundamental theorems of dimension theory, Bull. Acad. Polon. Sci. Ser. Sci. Math. 28 (1980), 595-602.

$\left[\mathrm{O}_{\mathbf{5}}\right] \ldots$, An inequality concerning three fundamental dimensions of paracompact $\sigma$-spaces, Proc. Amer. Math. Soc. 83 (1981), 790-792.

[Ok] A. Okuyama, Some generalizations of metric spaces, their metrization theorems and product spaces, Sci. Rep. Tokyo Kyoiku Daigaku Sect. A 9 (1967), 236-254.

[P] B. A. Pasynkov, On the spectral decomposition of topological spaces, Amer. Math. Soc. Transl. Ser. (2) 73 (1966), 83-134.

[Pe] A. R. Pears, Dimension theory of general spaces, Cambridge Univ. Press, Cambridge, 1975.

[S] F. G. Slaughter, The closed image of a metrizable space is $M_{1}$, Proc. Amer. Math. Soc. 37 (1973), 309-314.

[SN] F. Siwiec and J. Nagata, A note on nets and metrization, Proc. Japan Acad. 44 (1968), 623-627.

Department of Mathematics, Kanagawa University, RokKaku-bashi, Kanagawa-kU, YOKOHAMA, 221, JAPAN

Current address: Faculty of Education, Kagawa University, Saiwai-chō, Takamatsu, 760, Japan 